\title{
Investigating Organ Toxicity Profile of Tenofovir and Tenofovir Nanoparticle on the Liver and Kidney: Experimental Animal Study
}

\author{
Aniekan Imo Peter ${ }^{1}$, Edwin CS Naidu', Edidiong Akang ${ }^{1}$, Oluwatosin O Ogedengbe', \\ Ugochukwu Offor ${ }^{1}$, Sanjeev Rambharose ${ }^{2}$, Rahul Kalhapure ${ }^{2}$, Anil Chuturgoon ${ }^{3}$, \\ Thirumala Govender ${ }^{2}$ and Onyemaechi O Azu ${ }^{1,4}$ \\ 'Discipline of Clinical Anatomy, Nelson R. Mandela School of Medicine, University of KwaZulu-Natal, Durban, South \\ Africa \\ ${ }^{2}$ Discipline of Pharmaceutical Sciences, School of Health Sciences, University of KwaZulu-Natal, Durban, South Africa \\ ${ }^{3}$ Discipline of Medical Biochemistry, University of KwaZulu-Natal, Durban, South Africa \\ ${ }^{4}$ Department of Anatomy, School of Medicine, Windhoek, Namibia
}

\begin{abstract}
Tenofovir nanoparticles are novel therapeutic intervention in human immunodeficiency virus (HIV) infection reaching the virus in their sanctuary sites. However, there has been no systemic toxicity testing of this formulation despite global concerns on the safety of nano drugs. Therefore, this study was designed to investigate the toxicity of Tenofovir nanoparticle (NTDF) on the liver and kidney using an animal model. Fifteen adult male Sprague-Dawley (SD) rats maintained at the animal house of the biomedical resources unit of the University of KwaZulu-Natal were weighed and divided into three groups. Control animals (A) were administered with normal saline (NS). The therapeutic doses of Tenofovir (TDF) and nanoparticles of Tenofovir (NTDF) were administered to group B and C and observed for signs of stress for four weeks after which animals were weighed and sacrificed. Liver and kidney were removed and fixed in formal saline, processed and stained using H/E, PAS and MT stains for light microscopy. Serum was obtained for renal function test (RFT) and liver function test (LFT). Cellular measurements and capturing were done using ImageJ and Leica software 2.0. Data were analysed using graph pad 6, $p$ values $<0.05$ were significant. We observed no signs of behavioural toxicity and no mortality during this study, however, in the kidneys, we reported mild morphological perturbations widening of Bowman's space, and vacuolations in glomerulus and tubules of TDF and NTDF animals. Also, there was a significant elevation of glycogen deposition in NTDF and TDF animals when compared with control. In the liver, there were mild histological changes with widening of sinusoidal spaces, vacuolations in hepatocytes and elevation of glycogen deposition in TDF and NTDF administered animals. In addition to this, there were no significant differences in stereological measurements and cell count, LFT, RFT, weight changes and organo-somatic index between treatment groups and control. In conclusion, NTDF and TDF in therapeutic doses can lead to mild hepatic and renal histological damage. Further studies are needed to understand the precise genetic mechanism.
\end{abstract}

Key words: Tenofovir nanoparticles, Liver, Kidney, Histology

Correspondence to: Aniekan Imo Peter, Discipline of Clinical Anatomy, Nelson R. Mandela School of Medicine, University of KwaZulu-Natal, Durban 4013, South Africa

E-mail: petera@ukzn.ac.za

This is an Open-Access article distributed under the terms of the Creative Commons Attribution Non-Commercial License (http:// creativecommons.org/licenses/by-nc/3.0) which permits unrestricted non-commercial use, distribution, and reproduction in any medium, provided the original work is properly cited.

\begin{abstract}
Abbreviations: HIV, Human immunodeficiency virus; NTDF, Tenofovir Nanoparticle; TDF, Tenofovir; SD, Sprague-Dawley; H/E, Haematoxylin and Eosin; PAS, Periodic Acid Schiff; MT, Masson's Trichrome; LFT, Renal Function Test; RFT, Liver Function Test; HAART, Highly Active Antiretroviral Therapy; ART, Antiretroviral Therapy; NCs, Nano-carriers; HPMC, Hydroxypropylmethyl cellulose; NEs, Nanoemulsions; MGD, Mean Globule Diameter; PDI, Polydispersity Index; ZP, Zeta Potential; AIDS, Acquired Immunodeficiency Syndrome; LLA1E, Linolenic acid 1 Ester.
\end{abstract}




\section{INTRODUCTION}

Antiretroviral drugs have successfully reduced human immunodeficiency virus (HIV) infection from a death sentence to a chronic disease. Highly active antiretroviral therapy (HAART) improves health, prolongs life and has substantially reduced the risk of HIV transmission (1). The life expectancy of HIV-infected patients on antiretroviral therapy (ART) has remarkably improved and may approach those of uninfected population (2). However, there is growing concern about the potential for significant levels of drug resistance with expanded access to conventional antiretroviral drugs. Also, complete eradication of HIV from the body with current HIV treatment is a challenge to scientists and health practitioners. CD4+ T cells and macrophages in the lymphocytes act as latent reservoirs for HIV with the later serving as a host for viral genetic recombination producing mutant viral genes $(3,4)$.

Novel nanoparticle-based antiretroviral therapy (ART) delivery systems are vital in eradicating the virus from these reservoirs. They are more effective and efficient in HIV prevention and treatment (5). Nanoparticle-based delivery systems have been used not only to boost conventional treatments of HIV/AIDS; they have been used to advance therapeutic strategies: gene therapy, immunotherapy and vaccine developments (5). Nanotechnology-based platforms improve adherence to the drugs by keeping the circulation of drugs at therapeutic concentrations for longer durations and ensures that the dosage is kept simple. Targeted delivery of nano-carriers (NCs) to CD4 cells, macrophages, lymphoid tissue, and other tissues maximize ART delivery and effectiveness at latent sites (6).

Also, the unique physico-chemical properties and biomedical advances in the fabrication of NCs have provided remarkable applications in targeted drug delivery, diagnostics and patient compliance in the last decade (7-9). Despite this breakthrough, their clinical applications are challenged and limited by drug toxicity and bioavailability $(10,11)$.

The cellular toxicity of NCs depends on the type of biomaterial, size, shape, composition, surface charge (12), and surface chemistry (13).

There has been an upsurge of research in developing Tenofovir nanoemulsions for treatment of HIV infection in recent years. Improvement in permeation enhancement, such as nanoemulsions and nanogels, have led to more efficiency in drug delivery (14-17). However, the dearth of literature on in vivo studies on the systemic toxicity of any of these Tenofovir formulations is a challenge in achieving the therapeutic potential of these novel drugs. Only a few local toxicity studies on transdermal skin effect (17) vaginal routes $(18,19)$ and invitro studies $(16)$ were reported. Tenofovir nanoparticles (NTDF) with novel dendritic prop- erties have been developed by our team, invitro toxicity evaluation of the lipid component showed promising results (20). NTDF has potential to revolutionize the treatment of HIV infection through its effective penetration, reduction in administration frequency and effectiveness in attacking latent HIV virus in restricted areas in the body. However, safety concern in the biological system is a challenged that should be addressed. The aim of our present study was to investigate the histomorphology and biochemical effects of NTDF on the kidney and liver using SD rat model.

\section{MATERIALS AND METHODS}

Fifteen adult male Sprague-Dawley rats were maintained at the animal house of the biomedical resources unit, University of KwaZulu-Natal, South Africa was used for this study. The animals receive humane care in accordance with the principle of laboratory animal care of the national medical research council and the guide for the care and use of laboratory animals of the national academy of sciences (21). Ethical approval was obtained from the animal ethics committee of the University (AREC/ 010/016PP). All the rats were housed in well ventilated plastic cages ( 5 rats per cage) having dimensions of $(52 \mathrm{~cm}$ long $\times 36 \mathrm{~cm}$ wide and $24 \mathrm{~cm}$ high) and softwood shavings employed as beddings in the cages. They were maintained under standardized animal house conditions (temperature: $25^{\circ} \mathrm{C}$; light: approximately $12 \mathrm{hr}$ natural light per day) and were fed with standard rat pellets from (Meadow feeds a Division of Astral Operations Limited, Durban, South Africa) and given tap water ad libitum. The initial body weight of the animals was measured, and animals were randomly distributed with 5 rats/group.

TDF was procured from the Sinobright pharmaceutical company limited (Shanghai, China) Solutol HS 15®, PEG 400 , triethylamine and hydroxypropylmethyl cellulose (HPMC) were purchased from Sigma-Aldrich Co. Ltd (St. Louis, MO, USA). Milli-Q water purification system (Millipore corp., Burlington, MA, USA) was used to obtain purified water to prepare formulations.

Formulation of Nanoemulsions (NEs). NEs were prepared by an ultra-sonication method (22) using mixtures of linolenic acid ester (LLA1E) (20) as the oily phase, Solutol HS $15^{\circledR}$ and PEG 400 as the surfactant/cosurfactant mixture $\left(\mathrm{S}_{\mathrm{mix}}\right)$. The oily phase (LLA1E) and the $\mathrm{S}_{\text {mix }}$ were mixed, the required quantity of milli-Q water was added and sonicated [probe Omni Sonic Ruptor 400 Ultrasonic Homogenizer (OMNI International, Kennesaw, GA, USA)] at $30 \%$ amplitude for $10 \mathrm{~min}\left(20^{\circ} \mathrm{C}\right)$ to form the blank NE. Drug loaded NE was prepared following the above-mentioned method, except for the addition of $0.25 \%$ TDF to the LLAIE prior to mixing with the surfactant/co-surfactant mixture. No phase change was noted 
after addition of the drug or after equilibration. The NTDF were characterized for mean globule diameter (MGD), polydispersity index (PDI) and zeta potential (ZP) using a Zetasizer Nano ZS90 (Malvern Instruments Ltd., Worcestershire, UK) after suitably diluting with milli-Q water.
MGD is $129.1 \pm 3.3 \mathrm{~nm}$, PDI $0.192 \pm 0.04$ and ZP $20.9 \pm$ $2.0 \mathrm{mV}$.

Incorporation efficiency of tenofovir (TDF) in NE. The Tenofovir nanoemulsion was centrifuged at 4,500

Table 1. Weight changes in animals administered with TDF, NTDF and Normal saline

\begin{tabular}{lcrr}
\hline \hline Groups & Control \pm SEM $(\mathrm{g})$ & TDF \pm SEM $(\mathrm{g})$ & NTDF \pm SEM $(\mathrm{g})$ \\
\hline Initial body weight & $230 \pm 12.00$ & $220 \pm 7.00$ & $240 \pm 6.10$ \\
Final body weight & $256 \pm 8.30$ & $244 \pm 6.80$ & $262 \pm 6.50$ \\
Body weight differences & $20.40 \pm 2.10$ & $26.80 \pm 8.50$ & $25.60 \pm 0.90$ \\
Relative weight of kidney & $0.60 \pm 0.03$ & $0.60 \pm 0.02$ & $0.60 \pm 0.04$ \\
Relative weight of liver & $3.90 \pm 0.11$ & $3.60 \pm 0.13$ & $3.70 \pm 0.03$ \\
\hline
\end{tabular}

No significant differences at $p<0.05$.

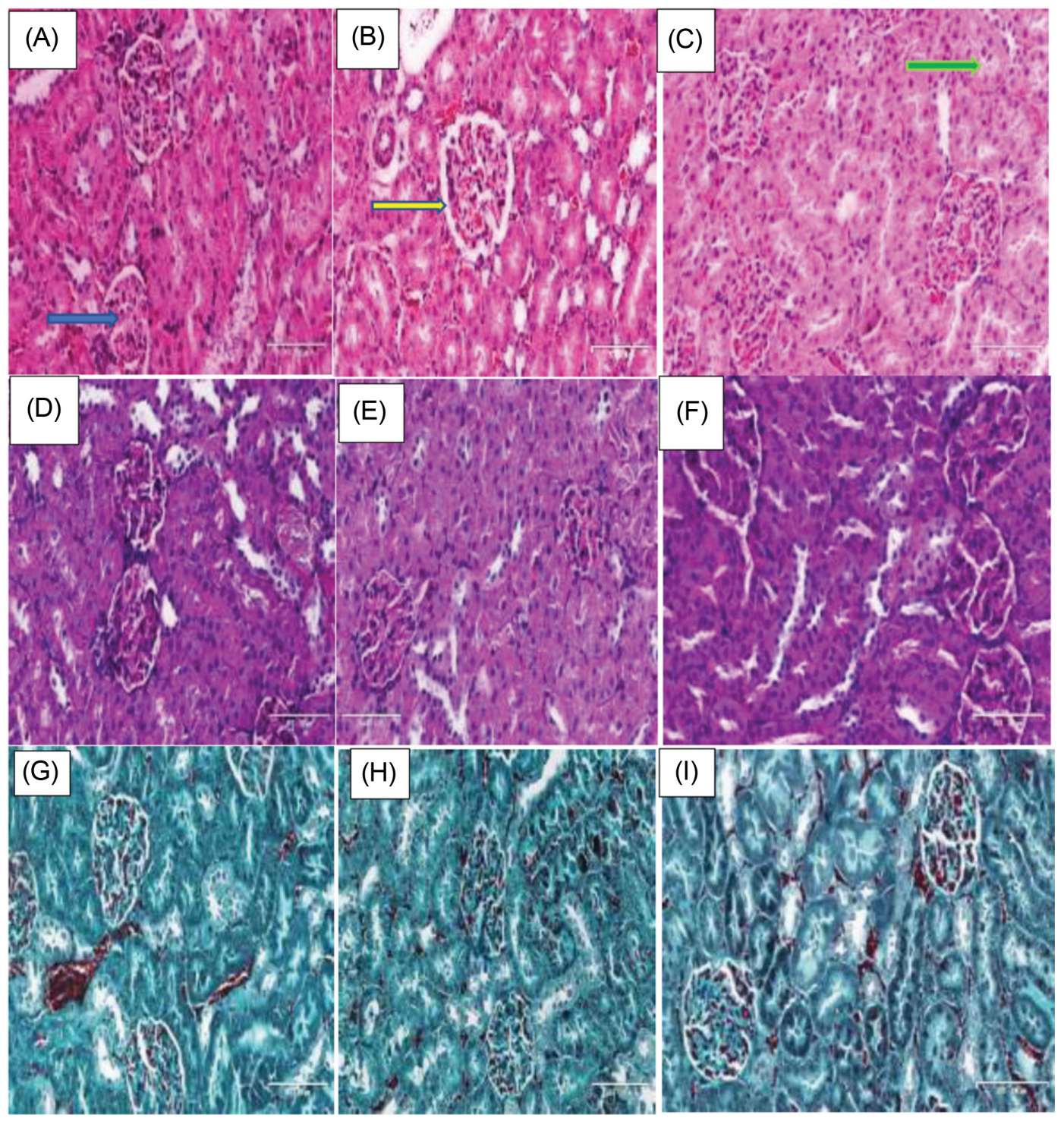

Fig. 1. Histological and stereological changes in Kidneys. (A-C) Animals stained with H/E, (D-F) PAS, and (G-I) Masson's trichrome. Blue arrow showing glomerulus, yellow arrow Bowman's space, and green arrow Proximal convoluted tubule. 
rpm and $4^{\circ} \mathrm{C}$ for 40 min using a Hermle Z326k centrifuge (HERMLE, Wehingen, Germany) to separate the unincorporated drug. The supernatant was analyzed at a $\lambda_{\max }$ of $262 \mathrm{~nm}$ using UV Spectrophotometer 1650 (Shimadzu, Kyoto, Japan) to determine the amount of unincorporated drug $\left(\mathrm{W}_{1}\right)$ from the total amount of drug used $\left(\mathrm{W}_{2}\right)$, the total drug content was estimated by dissolving the lipid emulsion in methanol. The percentage incorporation efficiency (\% IE) was calculated using the following equation:

$$
\% \mathrm{IE}=\frac{\mathrm{W}_{2}-\mathrm{W}_{1}}{\mathrm{~W}_{2}} \times 100
$$

and was estimated at $91.94 \pm 0.84 \%$ in this study.

- Experimental design: Fifteen male adult SD rats were randomly divided into 3 groups: Control animals (A) were administered with normal saline (NS). The therapeutic doses of TDF (4.3 mg/kg) and NTDF (4.3 mg/kg) were administered to group $\mathrm{B}$ and $\mathrm{C}$.

The experiment was conducted between 8:00 a.m. and 10:00 a.m. for a period of 4 weeks and all administrations were done via the intraperitoneal route.

\section{- Behavioural observation for signs of toxicity and} mortality: Animals were observed daily for signs of toxicity and for any mortality during the duration of the study. The observations included: feeding, grooming, pain, unusual sounds, aggression, distress, discomfort, convulsion, seizures, loss of consciousness. The record was updated on daily bases.

- Weight determination: Animals were weighed on the first day of the experiment, thereafter weekly and then on the last day of the experiment. Weights were taken in the morning between 8:00 and 10:00 a.m. Weights were taken using an electronic balance (Zeiss (Pty) Ltd. Gottingem, West Germany).

- Animal sacrifice and collection of samples: At end of the experiment, the animals were euthanized by placing them for $10 \mathrm{sec}$ in Halothane flask following which blood samples were collected via trans-cardiac puncture. Five milliliters $(5 \mathrm{~mL})$ of blood sample/rat was obtained into in a plain bottle and the serum obtained was centrifuged at 3,000 rpm for $10 \mathrm{~min}\left(\mathrm{~g}=9.78 \mathrm{~m} / \mathrm{s}^{2}\right)$.
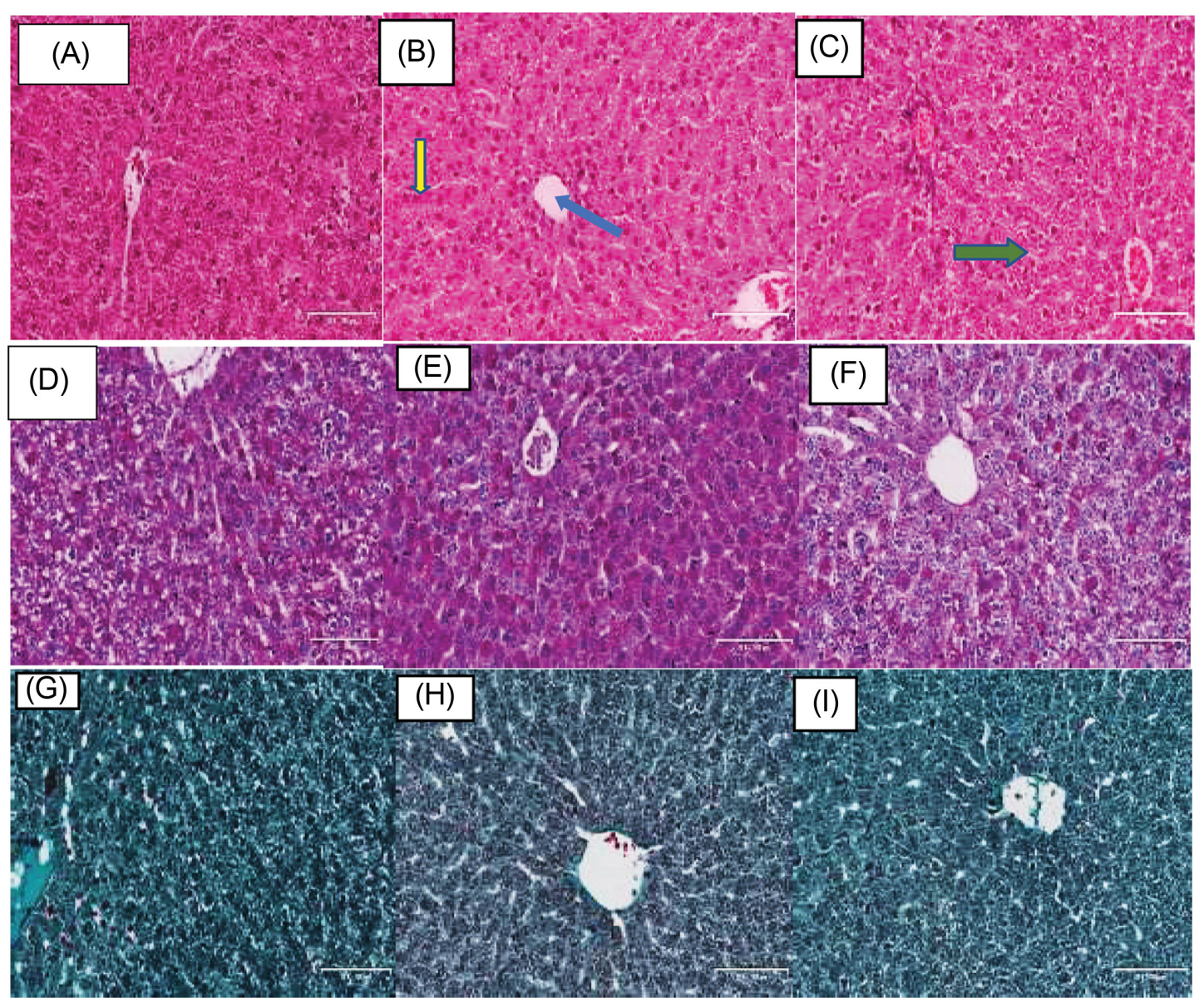

Fig. 2. Histological and stereological changes in liver. (A-C) Animals stained with H/E, (D-F) PAS, and (G-I) Masson's trichrome. Green arrow showing hepatocyte plate (Fig. 3B), yellow arrow showing sinusoidal space and blue arrow showing central vein. 
The kidney and liver were excised and weighed individually using an electronic balance (Mettler Toledo; Microstep (Pty) Ltd., Greifensee, Switzerland). 10\% formal saline fluid for histological analysis.

- Relative organ weight: The relative organ weights were calculated for the kidney and liver using the following formula:

$$
\text { (organ weight) } /(\text { total body weight }) \times 100
$$

- Histomorphometrical studies: Kidney and liver tissues were fixed in $10 \%$ formal saline. Samples were transferred to $70 \%$ ethanol. They were then processed using a graded ethanol series and embedded in paraffin. The paraffin sections were cut into $5 \mu \mathrm{M}$-thick slices using a microtome (microm HM 315 microtome, Walldorf, Germany) and stained with hematoxylin and eosin (H\&E), Periodic Acid Schiff (PAS) and Masson's trichrome (MT), studied under the microscope. Leica Microsystem was used to scan and snap the slides.

Stereological measurements for histomorphometric analysis, seven vertical sections were sampled as an unbiased numerical estimation of the diameter and cross-sectional area of the kidney (23). The cell count was done using systematic random sampling fair distribution from these seven sections. Eighteen Bowman's capsules and renal tubules were randomly selected from the slides. The vertical and horizontal diameters of each tubule and Bowman's capsule were measured as $\mathrm{d} 1$ and $\mathrm{d} 2$ respectively and the mean diameter (D) was recorded as an observation. The area of each Bowman's capsule was measured as (A), while the area of each tubule was measured as A1 and $\mathrm{A} 2(\mathrm{~A} 2$ = lumen of tubule) respectively area of the tubule (A) was calculated as (AI-A2). Slides were scanned using Leica SCN 400 (Leica Microsystems GmbH, Wetzlar, Germany) and measurements were taken using the image analyzer and Leica microsystem software, Leica Microsystems $\mathrm{GmbH}$ ) cell count and colour intensity were calculated through ImageJ cell software (National Insitute of Health, Bethesda, MD, USA). Analysis of results was by standard statistical package graph pad 6 values of $p<0.05$ were regarded as significant.

\section{RESULTS}

Behavioural observations for signs of toxicity and mortality. There were no stress symptoms in the animals for the duration of the study and there was no mortality.

Changes in weight and organo-somatic index. Body weight differences and organo-somatic index of kidney and the liver were not significant (Table 1).

Histological and stereological changes. H\&E sections of the kidney showed mild morphological perturba-
Table 2. Stereological measurements of the Bowman's Capsular Diameter, Bowman's capsular Area, Bowman's capsular space, Proximal convoluted tubular diameter and area. Distal convoluted tubular diameter and area of the kidney

\begin{tabular}{lccc}
\hline \hline Parameters & Control & TDF & NTDF \\
\hline BMC-A & $5900 \pm 450$ & $6600 \pm 300$ & $5900 \pm 440$ \\
BMC-D & $89 \pm 3.80$ & $92 \pm 1.80$ & $89 \pm 3.80$ \\
BMC-S & $6.1 \pm 0.40$ & $6.8 \pm 0.35$ & $6.3 \pm 0.37$ \\
PCT-D & $46 \pm 23$ & $49 \pm 17$ & $45 \pm 12$ \\
PCT-A & $1500 \pm 85$ & $1700 \pm 150$ & $1400 \pm 75$ \\
DCT-D & $35 \pm 1.70$ & $40 \pm 1.50$ & $40 \pm 1.50$ \\
DCT-A & $1200 \pm 64$ & $1300 \pm 130$ & $1300 \pm 110$ \\
\hline
\end{tabular}

BMC-A Bowman's Capsular area, BMC-D Bowman's capsular diameter, BMC-S Bowman's capsular space, PCT-D Proximal convoluted tubular diameter BMC-A Proximal convoluted tubular area, DCT-D Distal convoluted tubular diameter, DCT-A Distal convoluted tubular area.

No significant differences at $p<0.05$.

tions with widening of Bowman's space, and vacuolations in the photomicrograph of TDF and NTDF animals. NTDF animals look better than TDF group (Fig. 1A-1C).

In the liver, there were mild morphological/ histological changes with a widening of sinusoidal spaces, and vacuolations in TDF and NTDF animals which mirrored the changes in the kidney (Fig. 2A-2C).

Stereology. Stereological measurements showed no significant changes in Bowman capsule diameter, Bowman's capsule area, Bowman's space, Proximal convoluted tubular diameter PCTD, Proximal convoluted tubular area, distal convoluted tubular diameter, Distal convoluted tubular area between the control and treatment group $\mathrm{B}$ and $\mathrm{C}$ (Table 2).

- PAS and MT staining intensity: In the kidney, we reported a significant elevation of intensity in TDF animals (120 \pm 8.0 pixels) animals when compared with NTDF (94 \pm 13.0 pixels) and controls $(97 \pm 6.3$ pixels $)$ in PAS stain (Fig. 1D-1F, Table 3). There was also increased of intensity in MT stain of both the NTDF $(130 \pm 3.2$ pixels) and TDF (140 \pm 4.7 pixels) groups when compared with controls $(110 \pm 9.2$ pixels $)$ (Fig. 1G-1I, Table 3$)$.

In the liver, we reported a significant elevation of inten-

Table 3. Intensity of Staining in Kidney and liver (PAS and MT in Pixels)

\begin{tabular}{llrcr}
\hline \hline Test & Organ & Control & \multicolumn{1}{c}{ TDF } & \multicolumn{1}{c}{ NTDF } \\
\hline PAS & Kidney & $97 \pm 6.3$ & $120 \pm 8.0^{*}$ & $94 \pm 13.0$ \\
PAS & Liver & $87 \pm 6.5$ & $112 \pm 7.1^{*}$ & $92 \pm 13.2$ \\
MT & Kidney & $110 \pm 9.2$ & $140 \pm 4.7^{*}$ & $130 \pm 3.2^{\dagger}$ \\
MT & Liver & $130 \pm 5.1$ & $130 \pm 10.0$ & $140 \pm 10$ \\
\hline
\end{tabular}

"Significantly higher than control and NTDF group.

${ }^{\dagger}$ Significantly higher than control $p<0.05$. 
(A)

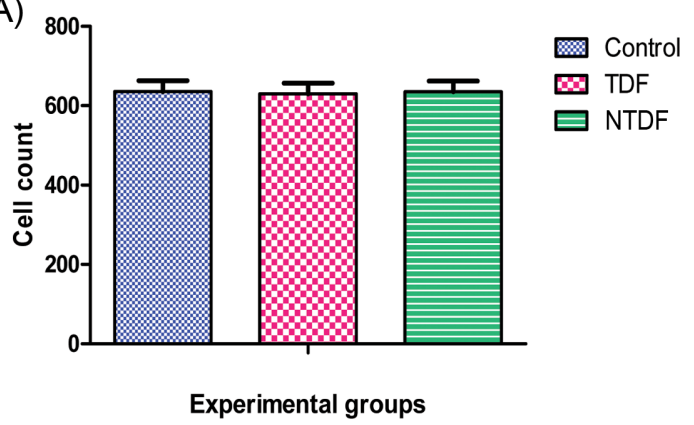

(B)

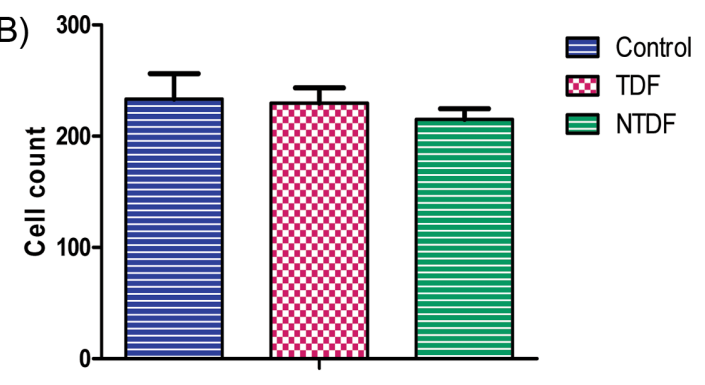

Experimental groups

Fig. 3. Cell count. (A) Renal cell count, (B) Liver cell count.

(A)

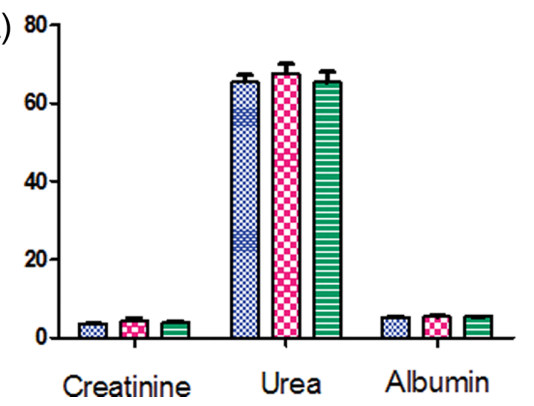

(B)

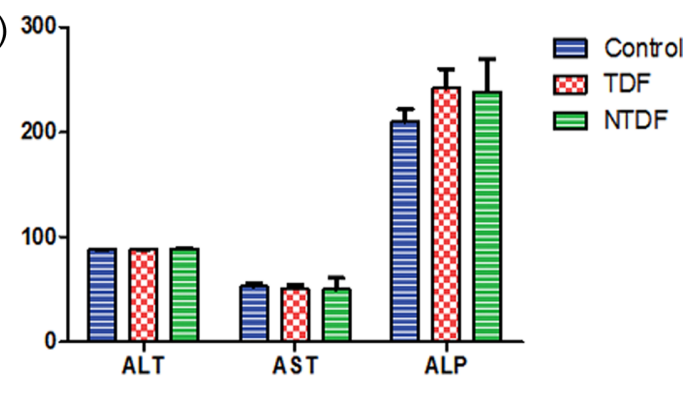

Fig. 4. Biochemical changes in serum. (A) Serum levels of Urea, creatinine levels and albumin, (B) Serum levels of alanine transaminase (ALT), aspartate transaminase (AST) and alkaline phosphatase (ALP).

sity in TDF animals (112 \pm 7.1 pixels) when compared with NTDF group $(92.1 \pm 13.2$ pixels) and controls $(86.6 \pm$ 6.2 pixels) in PAS stain (Fig. 2D-2F, Table 3). There was no significant difference of intensity in MT stain of both the NTDF $(130 \pm 5.1$ pixels $)$ and TDF $(130 \pm 10.0$ pixels $)$ and controls (140 \pm 10.0 pixels) (Fig. 2G-2I, Table 3).

Cell count. In cell count, there was no significant difference between treatment group and control in the kidney and liver. Though there was a slight decline in the treatment groups (Fig. 3).

Biochemical changes in serum. There were no significant changes in serum levels of Urea, creatinine levels, and albumin. We also reported no significant changes in serum levels of alanine transaminase (ALT), aspartate transaminase (AST) and alkaline phosphatase (ALP) in the liver (Fig. 4).

\section{DISCUSSION}

HIV is still a major public health issue in South Africa affecting young people mostly in their prime and productive age (24). Globally, since the start of the epidemic, an estimated 78 million people have become infected with HIV and 35 million people have died of AIDS-related illnesses $(25,26)$.
The dual problem of limitations of conventional ART in crossing biological barriers and drug resistance has encouraged innovation in new therapeutic approach in HIV treatment. Nano-drugs are novel innovations in the management of Human immunodeficiency virus (HIV) pandemic, especially resistant strains of the virus in their sanctuary sites. However, nanoparticles have potentials for toxicity (27). The global concern on the safety of nanoparticles must be addressed to achieve the full potential of this new drug delivery system. Toxicity testing of new compounds is essential for drug development process. The preclinical toxicity testing on various biological systems reveals the species-, organ- and dose-specific toxic effects of an investigational product. The toxicity of substances can be observed by in vitro studies using cells/cell lines and in vivo exposure on experimental animals (28). Our study was designed to investigate toxic effects of NTDF on behavioural changes, blood chemistry and histology of kidney and liver using animal model.

We reported no signs of behavioural changes, weight changes nor mortality for the 4 weeks of drug administration across the groups. This may imply that the drug has low toxicity. The adverse effects which are used to assess toxicity range from the very mild non-clinical signs of lethargy or effects on weight, to a more substantial effect such as convulsion. Behavioural changes are the critical tool in toxicity testing as animals should be protected from 
stress and pain (29). An example of drug adverse effect and toxicity can be seen in neuroleptics which are efficacious but they lead to behavioural toxicity of extrapyramidal effects, sedation and weight gain (30). Organ to body weight index which is a key index in drug toxicity determination was not different between the treatment group and control group. Analysis of organ weight in toxicology studies is an important factor for identification of potentially harmful effects of drugs $(31,32)$.

Also, we noted mild histological perturbations in the kidney and liver in the TDF and NTDF animals, when compared with the control. These changes were mirrored by changes in collagen deposition and fibrosis as demonstrated by staining intensity of PAS and MT staining. There was significant elevation of PAS in the TDF and significant elevation of MT in both TDF and NTDF groups when compared with controls. This implies that there was significant level of collagen deposition and fibrosis due to these drugs $(23,33,34)$. The kidney and liver are vital organs in drug metabolism, as such will give a better assessment of drug toxicity (35-37). The stereological measurements and cell count were not significantly different between the treatment groups (TDF, NTDF) and control. A previous study had reported low toxicity profile for lipid based nanoparticles (38). Another research investigating the cytotoxicity of iron oxide nanoparticles in vivo and in vitro studies reported low toxicity in the liver and kidney (39).

Biochemical markers play an important role in accurate diagnosis and for assessing risk and adopting therapy that improves clinical outcome. Over decades research and utilization of biomarkers had evolved substantially and creatinine and urea are commonly used as markers of kidney function. The creatinine clearance test is used to monitor the progression of renal disease (40). We reported no significant difference in urea and creatinine level across all the groups in variance with another research which reported significant renal toxicity of nanoparticles (41).

Liver disease is often reflected by biochemical abnormalities of liver function. AST and ALT can serve as markers of hepatocellular injury (42). In our study AST, ALT, ALP and albumin levels were not significantly different from control. The adverse effects of substances on animal physiology can range from minor changes, such as reduced weight gain, small physiological alterations, changes in the levels of circulating hormones, to severe effects such as organ function loss leading to death.

In summary, we observed no signs of behavioural toxicity and no mortality in this study, however, there were mild histological alterations in the liver and kidney. We reported no alteration in renal function test and liver function test between NTDF, TDF and control animals. Also, the weight, organo-somatic index and stereology measure- ments were not significantly different.

In conclusion, NTDF and TDF used in this protocol resulted in mild hepatic and renal histological damage. Further studies are needed to unravel the genetic mechanism of injury.

\section{ACKNOWLEDGMENTS}

The authors acknowledge the College of Health Science, University of KwaZulu-Natal for Operational funds to AIP and ECSN. TG and OOA were supported via the Nano-mobility grant and OOA is also supported by South African National Research Foundation grant U99053. We also acknowledge the technical support from Dr. Sanil Singh and Dr. Linda Bester of Biomedical Research Unit, University of KwaZulu-Natal.

\section{CONFLICT OF INTEREST}

Financial arrangement (funding: OOA: NRF CPRR (U99053) TG \& OOA: CHS-UKZN Nano-mobility grant).

Received February 5, 2018; Revised April 3, 2018; Accepted May 8, 2018

\section{REFERENCES}

1. Desai, M., Iyer, G. and Dikshit, R. (2012) Antiretroviral drugs: critical issues and recent advances. Indian J. Pharmacol., 44, 288-298.

2. Johnson, L.F., Mossong, J., Dorrington, R.E., Schomaker, M., Hoffmann, C.J., Keiser, O., Fox, M.P., Wood, R., Prozesky, H., Giddy, J., Garone, D.B., Cornell, M., Egger, M. and Boulle, A. (2013) Life expectancies of South African adults starting antiretroviral treatment: collaborative analysis of cohort studies. PLoS Med., 10, e1001418.

3. Otieno, M. (2015) Why novel nanoparticle-based delivery platforms hold key for HIV/AIDS treatment and prevention? HIV/AIDS Res. Treat. Open J., 2, 81-85.

4. Richman, D.D., Margolis, D.M., Delaney, M., Greene, W.C., Hazuda, D. and Pomerantz, R.J. (2009) The challenge of finding a cure for HIV infection. Science, 323, 1304-1307.

5. Mamo, T., Moseman, E.A., Kolishetti, N., Salvador-Morales, C., Shi, J., Kuritzkes, D.R., Langer, R., von Andrian, U. and Farokhzad, O.C. (2010) Emerging nanotechnology approaches for HIV/AIDS treatment and prevention. Nanomedicine (Lond.), 5, 269-285.

6. Parboosing, R., Maguire, G.E., Govender, P. and Kruger, H.G. (2012) Nanotechnology and the treatment of HIV infection. Viruses, 4, 488-520.

7. Liu, J., Huang, Y., Kumar, A., Tan, A., Jin, S., Mozhi, A. and Liang, X.J. (2014) pH-sensitive nano-systems for drug delivery in cancer therapy. Biotechnol. Adv., 32, 693-710.

8. Zhang, Q., Wang, X., Li, P.Z., Nguyen, K.T., Wang, X.J., Luo, Z., Zhang, H., Tan, N.S. and Zhao, Y. (2014) Biocompatible, uniform, and redispersible mesoporous silica nanoparti- 
cles for cancer targeted drug delivery in vivo. Adv. Funct. Mater., 24, 2450-2461.

9. Sohail, M.F., Sarwar, H.S., Javed, I., Nadhman, A., Hussain, S.Z., Saeed, H., Raza, A., Bukhari, N.I., Hussain, I. and Shahnaz, G. (2017) Cell to rodent: toxicological profiling of folate grafted thiomer enveloped nanoliposomes. Toxicol. Res., 6, 814-821.

10. Shi, J., Votruba, A.R., Farokhzad, O.C. and Langer, R. (2010) Nanotechnology in drug delivery and tissue engineering: from discovery to applications. Nano Lett., 10, 32233230.

11. Yoo, J.-W., Irvine, D.J., Discher, D.E. and Mitragotri, S. (2011) Bio-inspired, bioengineered and biomimetic drug delivery carriers. Nat. Rev. Drug Discov., 10, 521-535.

12. Daglar, B., Ozgur, E., Corman, M., Uzun, L. and Demirel, G. (2014) Polymeric nanocarriers for expected nanomedicine: current challenges and future prospects. RSC $A d v$. 4, 4863948659.

13. Zoroddu, M.A., Medici, S., Ledda, A., Nurchi, V.M., Lachowicz, J.I. and Peana, M. (2014) Toxicity of nanoparticles. Curr. Med. Chem., 21, 3837-3853.

14. Suvarna, V.M. and Sangave, P.C. (2017) Development and validation of stability indicating rp-hplc method for tenofovir solid lipid nanoparticles. IJPSR, 8, 658-666.

15. Matlhola, K., Katata-Seru, L., Tshweu, L., Bahadur, I., Makgatho, G. and Balogun, M. (2015) Formulation and optimization of Eudragit RS PO-tenofovir nanocarriers using Box-Behnken experimental design. J. Nanomater, 2015, 630690.

16. Alukda, D., Sturgis, T. and Youan, B.C. (2011) Formulation of tenofovir-loaded functionalized solid lipid nanoparticles intended for HIV prevention. J. Pharm. Sci., 100, 33453356.

17. Rambharose, S., Kalhapure, R.S. and Govender, T. (2017) Nanoemulgel using a bicephalous heterolipid as a novel approach to enhance transdermal permeation of tenofovir. Colloids Surf. B Biointerfaces, 154, 221-227.

18. Karim, Q.A., Karim, S.S.A., Frohlich, J.A., Grobler, A.C., Baxter, C., Mansoor, L.E., Kharsany, A.B., Sibeko, S., Mlisana, K.P., Omar, Z., Gengiah, T.N., Maarschalk, S., Arulappan, N., Mlotshwa, M., Morris, L. and Taylor, D. (2010) Effectiveness and safety of tenofovir gel, an antiretroviral microbicide, for the prevention of HIV infection in women. Science, 329, 1168-1174.

19. Mayer, K.H., Maslankowski, L.A., Gai, F., El-Sadr, W.M., Justman, J., Kwiecien, A., Mâsse, B., Eshleman, S.H., Hendrix, C., Morrow, K., Rooney, J.F. and Soto-Torres, L. (2006) Safety and tolerability of tenofovir vaginal gel in abstinent and sexually active HIV-infected and uninfected women. AIDS, 20, 543-551.

20. Rambharose, S., Kalhapure, R.S., Akamanchi, K.G. and Govender, T. (2015) Novel dendritic derivatives of unsaturated fatty acids as promising transdermal permeation enhancers for tenofovir. J. Mater. Chem. B, 3, 6662-6675.

21. National Research Council (1985) Guide for the Care and Use of Laboratory Animals. National academy of science, Washington, DC, USA.

22. Sonawane, S.J., Kalhapure, R.S., Jadhav, M., Rambharose, S., Mocktar, C. and Govender, T. (2015) Transforming lin- oleic acid into a nanoemulsion for enhanced activity against methicillin susceptible and resistant Staphylococcus aureus. RSC Adv., 5, 90482-90492.

23. Ogedengbe, O.O., Jegede, A.I., Onanuga, I.O., Offor, U., Naidu, E.C., Peter, A.I. and Azu, O.O. (2016) Coconut oil extract mitigates testicular injury following adjuvant treatment with antiretroviral drugs. Toxicol. Res., 32, 317-325.

24. Statistics SAoo (2016) Statistics South Africa. P0302. Midyear population estimates, 2016.

25. GBD 2015 HIV Collaborators (2016) Estimates of global, regional, and national incidence, prevalence, and mortality of HIV, 1980-2015: the global burden of disease study 2015. Lancet HIV, 3, e361-e387.

26. UNAIDS (2017) Fact sheet - Latest statistics on the status of the AIDS epidemic.

27. Yildirimer, L., Thanh, N.T., Loizidou, M. and Seifalian, A.M. (2011) Toxicology and clinical potential of nanoparticles. Nano Today, 6, 585-607.

28. Parasuraman, S. (2011) Toxicological screening. J. Pharmacol. Pharmacother, 2, 74-79.

29. Council, N.R. (2010) Guide for the Care and Use of Laboratory Animals. National Academies Press.

30. Valdovinos, M.G., Schroeder, S.R. and Kim, G. (2003) Prevalence and correlates of psychotropic medication use among adults with developmental disabilities: 1970-2000. Int. Rev. Res. Ment. Retard., 26, 175-220.

31. Bailey, S.A., Zidell, R.H. and Perry, R.W. (2004) Relationships between organ weight and body/brain weight in the rat: what is the best analytical endpoint? Toxicol. Pathol., 32, 448-466.

32. Piao, Y., Liu, Y. and Xie, X. (2013) Change trends of organ weight background data in sprague dawley rats at different ages. J. Toxicol. Pathol., 26, 29-34.

33. Street, J.M., Souza, A.C., Alvarez Prats, A., Horino, T., Hu, X., Yuen, P.S. and Star, R.A. (2014) Automated quantification of renal fibrosis with Sirius Red and polarization contrast microscopy. Physiol. Rep., 2, e12088.

34. Onyije, F., Ngokere, A., Ligha, A., Mgbere, O. and Avwioro, O. (2017) Computer-assisted image analysis in the diagnosis of gynaecological lesions: a quantitative and comparative investigation of haematoxylin-eosin with special dyes on tissue. J. Cancer Res. Pract., 4, 5-13.

35. Alomar, M.J. (2014) Factors affecting the development of adverse drug reactions. Saudi Pharm. J., 22, 83-94.

36. Anders, M. (1980) Metabolism of drugs by the kidney. Kidney Int., 18, 636-647.

37. Kuntzman, R., Mark, L., Brand, L., Jacobson, M., Levin, W. and Conney, A. (1966) Metabolism of drugs and carcinogens by human liver enzymes. J. Pharmacol. Exp. Ther, 152, 151-156.

38. Liu, L., Ye, Q., Lu, M., Chen, S.-T., Tseng, H.-W., Lo, Y.-C. and Ho, C. (2017) A new approach to deliver anti-cancer nanodrugs with reduced off-target toxicities and improved efficiency by temporarily blunting the reticuloendothelial system with intralipid. Sci. Rep., 7, 16106.

39. Ghasempour, S., Shokrgozar, M.A., Ghasempour, R. and Alipour, M. (2015) Investigating the cytotoxicity of iron oxide nanoparticles in in vivo and in vitro studies. Exp. Toxicol. Pathol., 67, 509-515. 
40. Gowda, S., Desai, P.B., Kulkarni, S.S., Hull, V.V., Math, A.A. and Vernekar, S.N. (2010) Markers of renal function tests. N. Am. J. Med. Sci., 2, 170-173.

41. Iavicoli, I., Fontana, L. and Nordberg, G. (2016) The effects of nanoparticles on the renal system. Crit. Rev. Toxicol., 46, 490-560.

42. Ahmed, S.N. and Siddiqi, Z.A. (2006) Antiepileptic drugs and liver disease. Seizure, 15, 156-164. 